\title{
Diffusion Tensor Image Registration Using Tensor Geometry and Orientation Features"
}

\author{
Jinzhong Yang ${ }^{1}$, Dinggang Shen ${ }^{1,2}$, Christos Davatzikos ${ }^{1}$, and Ragini Verma ${ }^{1}$ \\ ${ }^{1}$ Department of Radiology, University of Pennsylvania, Philadelphia, PA, USA 19104 \\ \{Jinzhong.Yang, Dinggang.Shen, Christos.Davatzikos, \\ Ragini.Verma\} @uphs . upenn.edu \\ ${ }^{2}$ Department of Radiology and Biomedical Research Imaging Center, \\ University of North Carolina, Chapel Hill, NC 27510 \\ dgshen@med.unc.edu
}

\begin{abstract}
This paper presents a method for deformable registration of diffusion tensor (DT) images that integrates geometry and orientation features into a hierarchical matching framework. The geometric feature is derived from the structural geometry of diffusion and characterizes the shape of the tensor in terms of prolateness, oblateness, and sphericity of the tensor. Local spatial distributions of the prolate, oblate, and spherical geometry are used to create an attribute vector of geometric feature for matching. The orientation feature improves the matching of the WM fiber tracts by taking into account the statistical information of underlying fiber orientations. These features are incorporated into a hierarchical deformable registration framework to develop a diffusion tensor image registration algorithm. Extensive experiments on simulated and real brain DT data establish the superiority of this algorithm for deformable matching of diffusion tensors, thereby aiding in atlas creation. The robustness of the method makes it potentially useful for group-based analysis of DT images acquired in large studies to identify disease-induced and developmental changes.
\end{abstract}

Keywords: Diffusion tensor imaging, structural geometry, tensor orientation, attribute vector, deformable registration.

\section{Introduction}

Diffusion tensor imaging (DTI) has emerged as a powerful and effective technique for analyzing the underlying white matter structure of brains [1]. DTI provides unique micro-structural and physiological insight into white matter tissue of brains, which in turn facilitates the study of development, aging, and disease on specific white matter regions of interest. In order to carry out group-based analysis and statistics, it is imperative to make different subjects comparable, thus requiring the spatial normalization of diffusion tensor (DT) images. However, spatial normalization of DT images is

* This work was supported by the National Institute of Health via grants R01-MH-070365 and R01-MH-079938. 
rendered challenging by the fact that the data representation is high dimensional and it requires not only the spatial warping, but also the tensor reorientation at each voxel $[2,3]$. Recent advances in DT image registration either employed a combination of different scalar maps derived from full tensor image for a multi-channel registration [4], or developed registration algorithms based on the full tensor similarity measurements [5, 6]. However, spatial normalization based on features extracted from full tensors has not been extensively researched yet. An earlier study applied oriented 3-D Gabor features extracted from tensors for matching [7], while a recent method employed major fiber bundles to align tensors[8]. Both methods demonstrate that registration can be improved in the white matter if features that characterize both tensor shape and orientation are used for matching with carefully chosen metrics.

In this paper, we apply tensor geometry and orientation features to DTI registration. We capitalize on the structural geometry of diffusion tensor [9] and develop a novel attribute vector consisting of geometric moments computed from the local spatial histograms of tensor geometric measures. This attribute vector is rotationally invariant, and integrates spatial information from local histograms computed at different scales. In order to improve the registration accuracy of white matter (WM) fiber tracts, we also incorporate the local statistical information of underlying fiber orientations for feature matching. These features provide richer anatomical information than merely using voxel's tensor by integrating anisotropy, shape, and orientation from an entire neighborhood of a voxel. We include these features into a hierarchical deformable registration technique on the lines of [10], to develop a deformable registration method for diffusion tensor images. Extensive experiments demonstrate the robustness and accuracy of DT image registration using these features.

\section{Methods}

Let $\lambda_{1} \geq \lambda_{2} \geq \lambda_{3} \geq 0$ be the three eigenvalues of a symmetric tensor $\mathbf{D}$, and $\hat{\mathbf{e}}_{i}$ be the normalized eigenvector corresponding to $\lambda_{i}$, then the tensor $\mathbf{D}$ can be denoted by

$$
\mathbf{D}=\lambda_{1} \hat{\mathbf{e}}_{1} \hat{\mathbf{e}}_{1}^{T}+\lambda_{2} \hat{\mathbf{e}}_{2} \hat{\mathbf{e}}_{2}^{T}+\lambda_{3} \hat{\mathbf{e}}_{3} \hat{\mathbf{e}}_{3}^{T} .
$$

Geometrically, tensor $\mathbf{D}$ can be represented by an ellipsoid with three axes oriented along its three eigenvectors, and three semi-axis lengths proportional to the square root of its three eigenvalues. Different shapes of the ellipsoid give rise to three geometric structures of diffusion tensors: prolate (linear) structure, in which diffusion is mainly in the direction corresponding to $\hat{\mathbf{e}}_{1}$; oblate (planar) structure, in which diffusion is restricted to a plane spanned by $\hat{\mathbf{e}}_{1}$ and $\hat{\mathbf{e}}_{2}$; and spherical structure with isotropic diffusion. Three geometric measures were proposed in [9] to describe how close the diffusion tensor is to the generic structures of prolateness, oblateness, and sphericity. They are respectively defined as

$$
c_{l}=\frac{\lambda_{1}-\lambda_{2}}{\lambda_{1}}, c_{p}=\frac{\lambda_{2}-\lambda_{3}}{\lambda_{1}}, c_{s}=\frac{\lambda_{3}}{\lambda_{1}} .
$$




\subsection{Tensor Geometric Feature for Matching}

A discriminative attribute vector is defined at each voxel from the geometric measures in (2). This attribute vector characterizes the local diffusion property by combining the local distributions of prolate, oblate, and spherical structures. For a specified voxel $v$, local histograms $\mathbf{h}_{l}(v)$ of $c_{l}, \mathbf{h}_{p}(v)$ of $c_{p}$, and $\mathbf{h}_{s}(v)$ of $c_{s}$ are computed from a spherical neighborhood region of voxel $v$ with a given appropriate radius $r$. These histograms roughly characterize the distribution of the tensor geometry in the neighborhood region. For each histogram, we compute its regular geometric moments as the statistical geometric features, i.e.

$$
m_{k}(v, n)=\sum_{i} i^{n} \mathbf{h}_{k}(v, i), \quad k=l, p, s ;
$$

where $\mathbf{h}_{k}(v, i)$ is the frequency of index $i$ in histogram $\mathbf{h}_{k}(v)$, and $m_{k}(v, n)$ is the $n$th order moment of this histogram. Low-order geometric moments are used to represent the geometric features for a histogram and form a vector as

$$
\mathbf{a}_{k}^{h i s t}(v)=\left\{m_{k}(v, n) \mid n=0,1,2\right\}, \quad k=l, p, s .
$$

In order to improve the accuracy of matching, we include the edge strength $b_{F A}^{\text {edge }}(v)$ of fractional anisotropy (FA) and the edge strength $b_{A D C}^{\text {edge }}(v)$ of apparent diffusion coefficient (ADC) into the attribute vector. These edge attributes are computed by a Canny edge detector [11] from FA and ADC scalar maps of DT image respectively. Therefore, the complete attribute vector at voxel $v$ can be represented as

$$
\mathbf{a}(v)=\left[\mathbf{a}_{l}^{h i s t}(v), \mathbf{a}_{p}^{h i s t}(v), \mathbf{a}_{s}^{h i s t}(v), b_{F A}^{\text {edge }}(v), b_{A D C}^{\text {edge }}(v)\right] .
$$

The attribute vector defined in (5) is rotationally invariant, which makes it attractive for registration. To make the feature vector more discriminative, the above attribute vector is computed at three different scales so that both global and local geometric features are accounted for. In each scale, the similarity of two attribute vectors, $\mathbf{a}(u)$ and $\mathbf{a}(v)$, of two points, $u$ and $v$, is defined as

$$
m(\mathbf{a}(u), \mathbf{a}(v))=\prod_{i}\left(1-\left|\mathbf{a}_{i}(u)-\mathbf{a}_{i}(v)\right|\right),
$$

where $\mathbf{a}_{i}(\cdot)$ is the $i$ th element in the attribute vector. Due to the redundancy between $c_{l}, c_{p}$, and $c_{s}$, we normally discard the $0^{\text {th }}$ and $1^{\text {st }}$ order geometric moments derived from $c_{p}$ when computing the similarity in (6).

We demonstrate the discrimination of the proposed attribute vector of geometric feature in Figs. 1 and 2 by comparing it with the FA feature [10] for diffusion tensor matching. Both points in major fiber tracts and small tracts have been examined. From the color-coded maps of similarities illustrated in Figs. 1 and 2, we can conclude that the geometric feature is much more discriminative than just using FA feature on both major and small fiber tracts (even on a single scale), with geometric feature being far superior on the smaller tracts. 


\subsection{Fiber Orientation Feature for Matching}

Properly aligning WM fiber tracts is a major concern in DTI registration. In order to further improve the registration accuracy of WM fiber tracts, we incorporate the local statistical information of underlying fiber orientations into the attribute vector defined in (5). The fiber orientation at voxel $v$ is approximated by the principal direction (PD) of tensor $\mathbf{D}$ weighted by the FA value at this voxel. Local spatial distribution of PD in the 3D space at voxel $v$, denoted by $\mathbf{H}_{P D}(v)$, can be estimated from the samples in a spherical neighborhood region with a radius $r$. The similarity of two points, $u$ and $v$, in terms of local PD distribution is characterized by entropy cross correlation (ECC) [12], a normalized form of mutual information, as

$$
E C C_{P D}(u, v)=2-2 \frac{E\left[\mathbf{H}_{P D}(u), \mathbf{H}_{P D}(v)\right]}{E\left[\mathbf{H}_{P D}(u)\right]+E\left[\mathbf{H}_{P D}(v)\right]},
$$

where $E$ denotes the joint or marginal differential entropy of the random variables of local PD distribution. The similarity with the orientation features at points $u$ and $v$ is then determined by the combination of Eqs. (6) and (7) as

$$
M(u, v)=m(\mathbf{a}(u), \mathbf{a}(v)) \cdot E C C_{P D}(u, v) .
$$

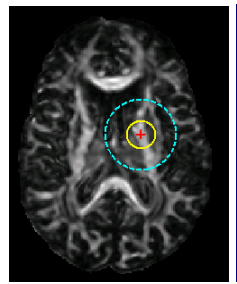

(a)

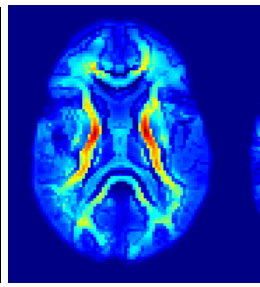

(b)

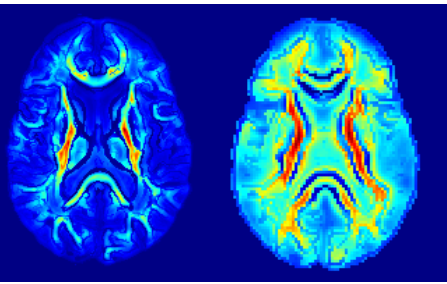

(c) (d)

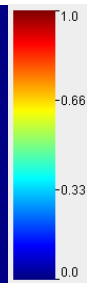

(e)

Fig. 1. Similarity of the points on major fiber tracts. The attribute vector of the crossed point in (a) is compared with the attribute vectors of other points in the image. (b) and (c) show the resulting map of similarities using the geometric feature computed at a coarse scale and a fine scale, respectively. (d) and (e) show the resulting map of similarities using the FA feature computed at a coarse scale and a fine scale, respectively. Red indicates high similarity.

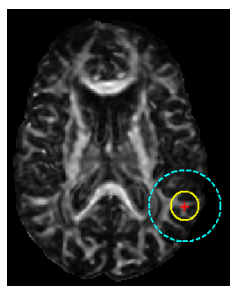

(a)

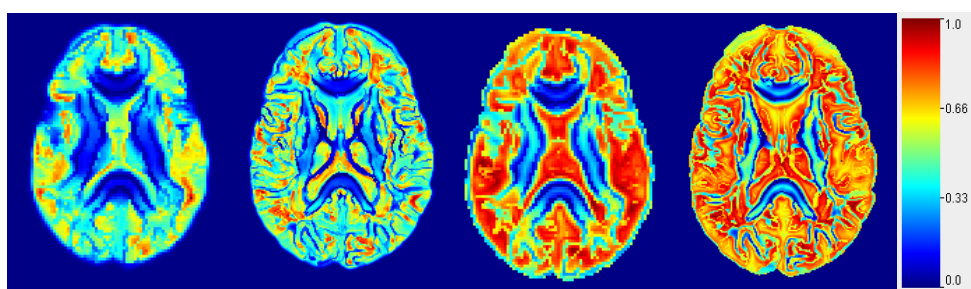

(b) (c) (e)

Fig. 2. Similarity of the points on small fiber tracts. Legends are the same as those in Fig. 1. 
$M(u, v)$ ranges from 0 to 1 where 1 indicates the most similar features. Since the PD of a tensor is meaningful only in high anisotropic anatomies such as WM fiber tracts, we consider the matching of orientation feature only for voxels with a high FA value.

\subsection{Deformable Registration with Geometry and Orientation Features}

The attribute vector of tensor geometry and orientation features described above is used in conjunction with the deformable techniques on the lines of the intensity histogram based HAMMER algorithm [10] to develop a DTI registration algorithm. In this algorithm, the input DT images are pre-registered linearly. In a first step, only the geometric feature is used for registration. Features are extracted once and not recalculated during registration, which requires features being rotationally invariant. Next, both tensor geometry and orientation features are included in registration. It serves to further refine the matching of WM fiber tracts. Due to the orientation feature applied, feature extraction is performed in each iteration and the tensors are warped and reoriented accordingly. The deformation field obtained as part of the spatial warping is used to determine the tensor reorientation, based on a spatially adaptive procedure that estimates the underlying fiber orientation [2], to produce properly reoriented tensors.

This algorithm employs a hierarchical structure to select distinct features, thus reducing ambiguity in finding correspondences. The edge strengths of FA and ADC maps are the criteria for choosing active points to drive the registration. In the initial stages of matching, only a few points with high edge strength are selected for matching in order to avoid local minima. As the matching progresses, more and more points with lower edge strengths become reliable and thus are selected to drive the registration. This hierarchical structure assists in achieving a robust and accurate registration.

\section{Results}

We have demonstrated the high matching accuracy of the geometric feature in different parts of the white matter fiber tracts in Figs. 1 and 2. In this section, we applied our method to register both human brains and mouse brains to demonstrate the efficiency of our method by comparing with two alternative deformable registration algorithms, intensity histogram based HAMMER [10] and Demons algorithm [13], when applied to FA maps. For the sake of simplicity and fairness, we compare FA featurebased registration with the geometric feature, establishing the superiority of the latter. In the next stage we also demonstrate that the orientation feature together with the geometric feature improves the matching of the WM fiber tracts.

\subsection{Matching Accuracy Comparison: Geometric Feature and FA Feature}

Ten simulated human brain DT images are generated by applying ten simulated deformation fields [14] to warp a real DT image that is regarded as the template. These ten simulated DT images are then registered back to the template space by our method using geometric feature and the intensity histogram based HAMMER using FA feature, respectively. The deformation errors between the registration results and the 
simulated ground truth are calculated for both methods. Fig. 3 shows the average registration error and the variance in each subject computed from the whole brain and WM fiber tracts, respectively. It demonstrates that using the geometric feature yields more accurate registration than using the FA feature, with respective population means as 0.89 voxels and 1.11 voxels for the whole brain. Comparing Figs. 3(a) and (b) shows the registration to be superior in the WM fiber tracts. The respective population means using geometric feature and FA feature are 0.75 voxels and 0.93 voxels in the regions with $\mathrm{FA}>0.25$.

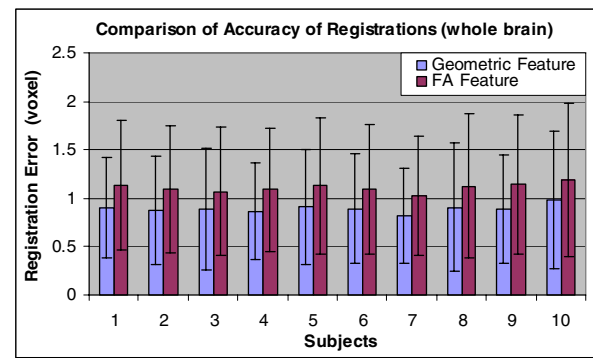

(a)

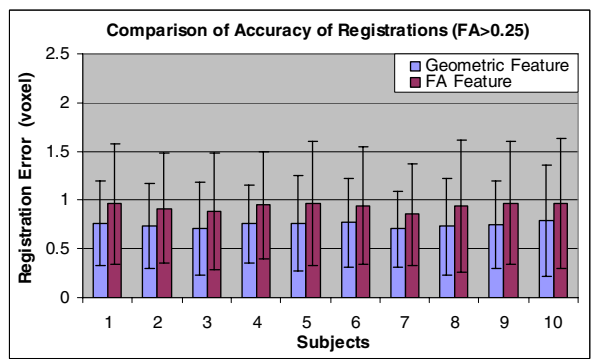

(b)

Fig. 3. Comparison of the registration accuracy using the geometric feature and FA feature. (a) shows the registration error computed from the whole brain, and (b) shows the registration error computed from WM fiber tracts with FA $>0.25$.

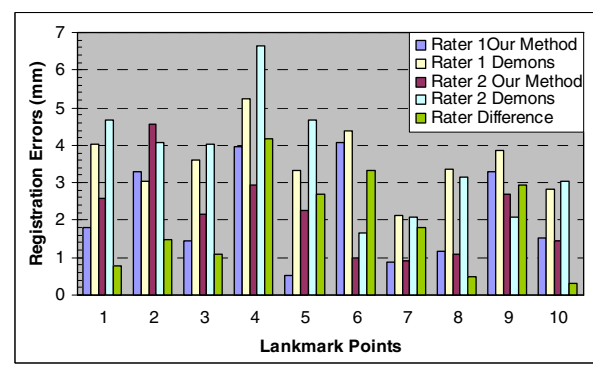

(a)

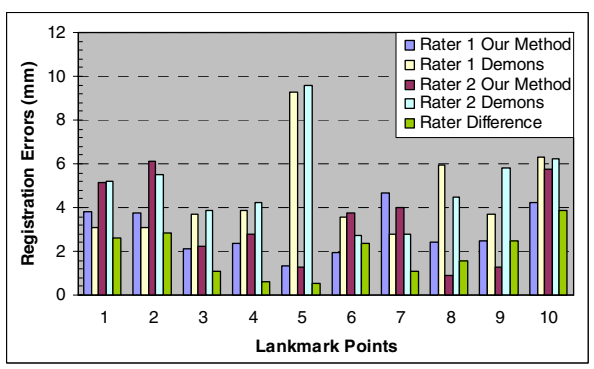

(b)

Fig. 4. Comparison of the registration errors on each of 10 landmark points identified by two raters on both major and minor WM fiber tracts, in two subjects (a) and (b), respectively

\subsection{DTI Registration of Human Brains}

To further demonstrate the efficiency of our method, we apply it to register real human brain DT images and compare it with the demons algorithm, which is implemented in ITK [15] and is applied to FA images. The same tensor reorientation scheme [2] is applied together with demons registration to produce the final warped DT image. Two subjects were registered to a template, each with voxel resolution as $0.9375 \times 0.9375 \times 2.5 \mathrm{~mm}$. 
We had two raters pick up 10 corresponding landmarks from each subject and template. These landmarks reside in both major and minor WM fibers and serve to evaluate the matching accuracy. For each pair of corresponding landmarks, we compute the registration errors for our proposed method and the demons algorithm. These results are shown in Fig. 4. We also show the variation between two raters as the "rater difference" in Fig. 4 for better understanding. Fig 4 illustrates that overall our method achieves more accurate and robust registration than the demons algorithm does. To visually observe the registration of WM fiber tracts, we demonstrate the overlaid WM fiber tracts in Fig 5. In Fig. 5, we compare the registered fiber tracts obtained from the demons algorithm, our method without adding the orientation feature described in Section 2.2, and our method with the added orientation feature. The results show that the registration with the orientation feature achieves the best matching of WM fiber tracts. The arrows point to those spurious fibers extracted from registered images, which might be resulted from inaccurate registration.

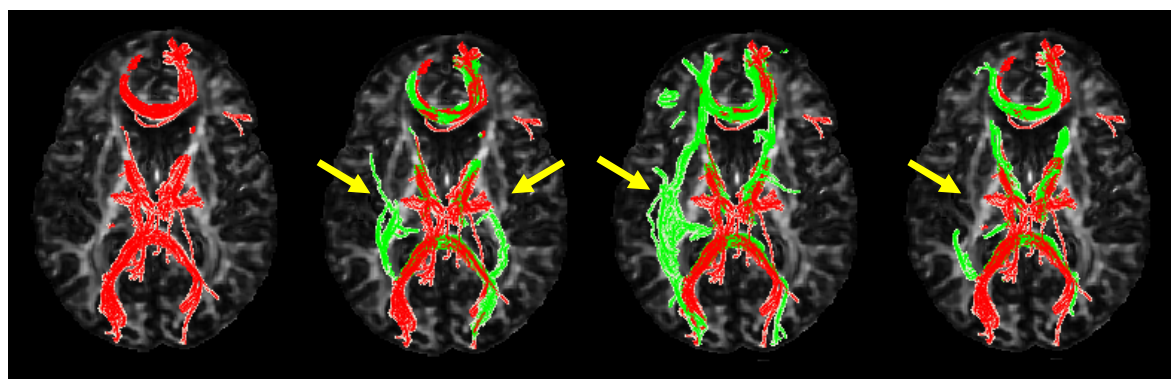

(a)

(b)

(c)

(d)

Fig. 5. Comparison of the overlaid WM fiber tracts in 3D space. (a) shows the WM fiber tracts extracted from the template. (b), (c), and (d) show the overlaid fiber tracts extracted from registered DT images by the demons algorithm, our method without adding orientation feature, and our method with added orientation feature, respectively.

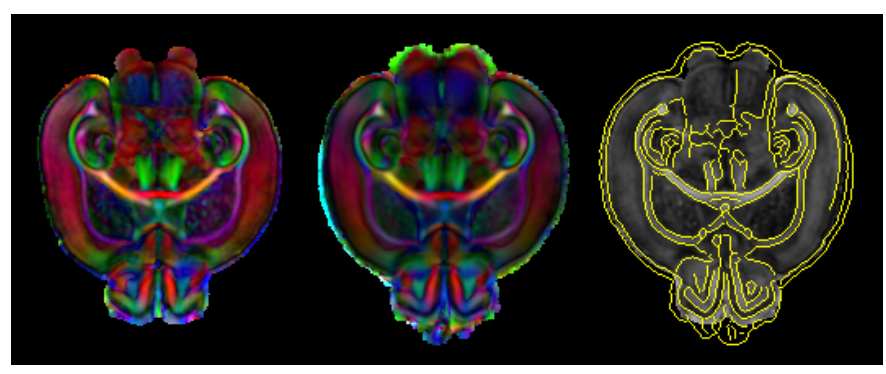

(a)

(b)

(c)

Fig. 6. Spatial normalization of 5 mouse brains. (a) Color map of the template. (b) Color map of the group averaged image. (c) Edges extracted from FA map of group averaged image superimposed on the FA map of template. 


\subsection{Creating an Atlas of Murine Brains}

We apply our method to spatially normalize 5 mouse brains scanned at Day 10 in order to create an atlas for this development stage by group-averaging the normalized DT images. One subject is identified as the template and the others are registered to it using the proposed method. The group-averaged image is computed by voxel-wise averaging the corresponding tensors in the individual warped subjects. Fig. 6 visually demonstrates the registration accuracy. The color map in Fig. 6 is the tensor PD weighted by the corresponding FA value. The color is encoded with green representing anterior-posterior, blue for feet-head, and red for left-right orientation. The sharpness of the average in Fig. 6(b) as compared to the template in Fig. 6(a), as well as the good matching of the edge map to the underlying FA image in Fig. 6(c), shows that a good spatial normalization has been achieved and even the thin tracts like internal and external capsules have been aligned well.

\section{Conclusions}

In conclusion, we have presented a novel attribute vector that characterizes the geometry and orientation of diffusion tensors and hence obtains superior matching and subsequent deformable registration. The features are incorporated into a hierarchical deformable registration algorithm, and the orientation feature improves the matching of the WM fiber tracts. The extensive experiments verified the efficiency of the features for matching of tensors and subsequently in obtaining a fully deformable registration framework for diffusion tensor images.

\section{References}

1. Pierpaoli, C., Jezzard, P., Basser, P.J., Barnett, A., Chiro, G.D.: Diffusion tensor MR imaging of human brain. Radiology 201(3), 637-648 (1996)

2. Xu, D., Mori, S., Shen, D., Zijl, P.C.M.v., Davatzikos, C.: Spatial Normalization of Diffusion Tensor Fields. Magnetic Resonance in Medicine 50(1), 175-182 (2003)

3. Alexander, D.C., Pierpaoli, C., Basser, P.J., Gee, J.C.: Spatial transformations of diffusion tensor magnetic resonance images. IEEE Transactions on Medical Imaging 20(11), 11311139 (2001)

4. Park, H.J., Kubicki, M., Shenton, M.E., Guimond, A., McCarley, R.W., Maier, S.E., Kikinis, R., Jolesz, F.A., Westin, C.-F.: Spatial Normalization of Diffusion Tensor MRI Using Multiple Channels. Neuroimage 20(4), 1995-2009 (2003)

5. Cao, Y., Miller, M., Mori, S., Winslow, R.L., Younes, L.: Diffeomorphic matching of diffusion tensor images. In: MMBIA, New York, NY, p. 67 (2006)

6. Zhang, H., Yushkevich, P.A., Alexander, D.C., Gee, J.C.: Deformable registration of diffusion tensor MR images with explicit orientation optimization. Medical Image Analysis 10(5), 764-785 (2006)

7. Verma, R., Davatzikos, C.: Matching of Diffusion Tensor Images Using Gabor Features. In: Proceedings of the IEEE International Symposium on Biomedical Imaging (ISBI), Arlington, Va, pp. 396-399 (2004) 
8. Ziyan, U., Sabuncu, M.R., O’Donnell, L.J., Westin, C.-F.: Nonlinear Registration of Diffusion MR Images Based on Fiber Bundles. In: Ayache, N., Ourselin, S., Maeder, A. (eds.) MICCAI 2007, Part I. LNCS, vol. 4791, pp. 351-358. Springer, Heidelberg (2007)

9. Westin, C.-F., Maier, S.E., Mamata, H., Nabavi, A., Jolesz, F.A., Kikinis, R.: Processing and Visualization of Diffusion Tensor MRI. Medical Image Analysis 6, 93-108 (2002)

10. Shen, D.: Image Registration by Local Histogram Matching. Pattern Recognition 40, 1161-1171 (2007)

11. Canny, J.: A computational approach to edge detection. IEEE Transactions on Pattern Analysis and Machine Intelligence 8(6), 679-698 (1986)

12. Huang, X., Sun, Y., Metaxas, D., Sauer, F., Xu, C.: Hybrid Image Registration based on Configural Matching of Scale-Invariant Salient Region Features. In: IEEE Workshop on Image and Video Registration, p. 167 (2004)

13. Thirion, J.P.: Non-rigid matching using demons. In: Proceedings of IEEE Conference on Computer Vision and Pattern Recognition, pp. 245-251 (1996)

14. Xue, Z., Shen, D., Karacali, B., Stern, J., Rottenberg, D., Davatzikos, C.: Simulating deformations of MR brain images for validation of atlas-based segmentation and registration algorithms. Neuro Image 33(3), 855-866 (2006)

15. ITK Software Guide, http: //www. itk.org/HTML/Documentation.htm 